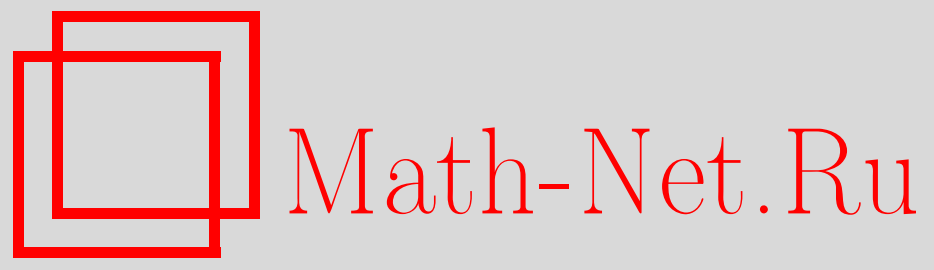

А. С. Холево, Асимптотическое оценивание параметра сдвига квантового состояния, Теория вероятн. и ее примен., 2004, том 49, выпуск 2, 335-350

DOI: https://doi.org/10.4213/tvp222

Использование Общероссийского математического портала Math-Net.Ru подразумевает, что вы прочитали и согласны с пользовательским соглашением

http://www . mathnet.ru/rus/agreement

Параметры загрузки:

IP: 3.85 .5 .30

26 апреля 2023 г., 05:19:53

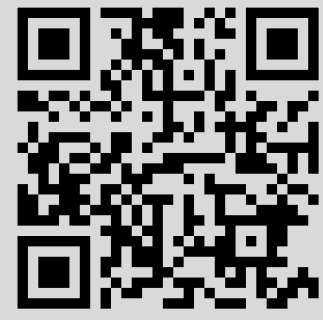




\title{
АСИМПТОТИЧЕСКОЕ ОЦЕНИВАНИЕ ПАРАМЕТРА СДВИГА КВАНТОВОГО СОСТОЯНИЯ ${ }^{1)}$
}

\begin{abstract}
Изучена асимптотика оптимальной ковариантной оценки параметра сдвига чистого квантового состояния. В регулярном случае, когда конечна дисперсия генератора группы сдвигов, рассматриваемая задача квантовой статистики оказывается связанной с энтропийным подходом к классической центральной предельной теореме, предложенным в 1959 году Ю.В. Линником. Рассмотрен и квантовый аналог оценивания параметра сдвига прямоугольного распределения, в котором условие регулярности нарушается. Продемонстрировано преимущество, обусловленное квантовым свойством сцепленности, в классе ковариантных оценок.
\end{abstract}

Ключевые слова и фразы: квантовая теория оценивания, ковариантные оценки, параметр сдвига, сцепленность.

1. Введение. Возникновение квантовой теории оценивания относится к концу 1960-х-1970-м гг. (см. [1], [2]). Первоначально она была предложена как метод постановки и решения задачи оптимального приема сигналов в квантовом канале связи. Впоследствии стало ясно, что идеи и методы квантовой теории оценивания дают адекватную концептуальную и математическую основу для формулировки и решения целого ряда проблем оснований квантовой теории и квантового измерения. В 1990-е гг. возникает новый интерес, стимулированный совершенствованием физического эксперимента и появлением идей квантовой криптографии и компьютинга, см., например, [3]-[8]. Речь идет о высокоточном, в идеале - оптимальном измерении параметров состояния элементарных квантовых объектов. Соответствующая квантовая теория оценивания концептуально следует классической, однако использует для описания основных понятий аппарат теории операторов (матриц), т.е. опирается на некоммутативную теорию вероятностей, см., например, [9].

Квантовая теория оценивания выявляет ряд необычных с классической точки зрения статистических свойств квантовых систем. Одно

* Математический институт им. В. А. Стеклова РАН, ул. Губкина, 8, 119991 Москва, ГСП-1, Россия; e-mail: holevo@mi.ras.ru

1) Работа частично поддержана грантами НШ 1758.2003.1, ИНТАС 00-738. 
из наиболее интересных отличий возникает при рассмотрении повторной независимой выборки из квантовой генеральной совокупности и состоит в наличии так называемых сцепленных (entangled) оценок, которые могут быть более эффективны, чем оценки, не обладающие этим свойством. Математически свойство сцепленности обусловлено тем, что составные квантовые системы, в отличие от классических, описываются тензорным, а не декартовым произведением, допускающим операцию суперпозиции. В настоящей статье этот феномен демонстрируется на простой, но важной модели асимптотического ковариантного оценивания параметра сдвига чистого квантового состояния. Эта задача является некоммутативным аналогом эквивариантного оценивания параметра сдвига классического распределения вероятностей [10] и в то же время дает ключ к пониманию проблемы канонической сопряженности и нестандартных соотношений неопределенностей (время-энергия, фаза-число квантов) в квантовой механике [2], [9].

Отметим, что чистые классические состояния - это вырожденные распределения вероятностей, для которых задача оценивания тривиальна. В квантовой статистике уже в этом случае задача ковариантного оценивания содержательна, а в случае параметра сдвига смешанного состояния она вообще остается нерешенной. Резкое возрастание сложности при переходе от чистых к смешанным состояниям является характерной особенностью задач квантовой статистики и теории информации.

Еще одно упрощение в рассматриваемой задаче - это одномерность оцениваемого параметра. При оценивании многомерного параметра в квантовом случае возникают дополнительные сложности и новые эффекты, связанные с требованием коммутативности (одновременной наблюдаемости) компонент оценки, которое в классическом случае выполняется автоматически. Это проявляется, в частности, в существовании нескольких несравнимых между собой матричных неравенств типа РаоКрамера [2].

Весьма интересной представляется неожиданная связь рассматриваемой здесь асимптотической задачи квантовой статистики и энтропийного подхода к классической центральной предельной теореме, предложенного Ю. В. Линником [11] и впоследствии усовершенствованного в работах [12], [13]. Результат последней работы позволяет дать окончательную формулировку для асимптотики дисперсии оптимальной ковариантной квантовой оценки при минимальных предположениях регулярности.

Настоящая работа является развитием заметки [14], в которой асимптотическая задача квантовой теории оценивания рассматривалась, повидимому, впервые. В п. 2 кратко напоминаются основные понятия квантового статистического описания, а в п. 3 - основные факты не- 
асимптотического ковариантного оценивания параметра сдвига. В п. 4 исследована структура оптимальной ковариантной оценки и показано, каким образом возникает сцепленность в квантовом аналоге схемы независимых повторных испытаний. В пп. 5, 6 исследуется регулярный случай, когда генератор группы сдвигов имеет конечную дисперсию в рассматриваемых состояниях. В п. 5 показано, что оптимальная ковариантная оценка асимптотически нормальна и достигает нижней границы, задаваемой соотношением неопределенностей. Пункт 6 посвящен асимптотическому сравнению оптимальной оценки с полуклассической ковариантной оценкой, не использующей сцепленность. В п. 7 рассматривается нерегулярный случай, аналогичный классическому оцениванию параметра сдвига прямоугольного распределения.

2. Состояния, наблюдаемые, моменты. Здесь кратко напоминаются основные понятия қвантового статистического описания (см. [2], [9]).

С каждой квантовомеханической системой связывается сепарабельное комплексное гильбертово пространство $\mathscr{H}$. Состояния системы описываются операторами плотности, т.е. эрмитовыми положительными операторами $S$ в $\mathscr{H}$ с единичным следом:

$$
S^{*}=S \geqslant 0 ; \quad \operatorname{Tr} S=1 .
$$

Крайние точки выпуклого множества состояний называются иистыми состояниями. Соответствующие операторы плотности - это всевозможные одномерные проекторы $S=|\psi\rangle\langle\psi|$, где $\psi$ - единичные векторы в $\mathscr{H}$ (здесь и далее используются обозначения Дирака, см., например, цитированные выше книги). Наблюдаемой со значениями в измеримом пространстве $(\mathscr{X}, \mathscr{B})$ называется мера $M$ на $\mathscr{B}$ со значениями в конусе эрмитовых положительных операторов в $\mathscr{H}, \sigma$-аддитивная в слабой операторной топологии и такая, что $M(\mathscr{X})=I$, где $I$ - единичный оператор в $\mathscr{H}$ (такие меры называются также разложениями единицы в $\mathscr{H}$ ). Распределение вероятностей наблюдаемой $M$ в состоянии $S$ задается формулой

$$
\mu_{S}^{M}(B)=\operatorname{Tr} S M(B), \quad B \in \mathscr{B} .
$$

Для нас здесь особый интерес будут представлять вещественные наблюдаемые, для которых $\mathscr{X}$ является измеримым подмножеством вещественной прямой R. В этом случае математическое ожидание (среднее значение) наблюдаемой $M$ в состоянии $S$ есть

$$
\mathbf{E}_{S}(M)=\int_{\mathscr{X}} x \mu_{S}^{M}(d x)=\operatorname{Tr} S A
$$

где $A=\int_{\mathscr{X}} x M(d x)$ - симметричный оператор. Эти соотношения имеют место во всяком случае для ограниченных наблюдаемых (для 
которых $\mathscr{X}$ - ограниченное множество). Для неограниченных наблюдаемых им можно придать математический смысл при дополнительных предположениях, например, конечности второго момента $\int_{\mathscr{X}} x^{2} \mu_{S}^{M}(d x)$ (см. [2]). Если $M$ - проекторнозначная мера (ортогональное разложение единицы), то $M$ - спектральная мера самосопряженного оператора $A$ и соответствие между $M$ и $A$ взаимно однозначно. В этом случае формула (3) переходит в статистический постулат Борна-фон Неймана и правая часть будет обозначаться также $\mathbf{E}_{S}(A)$.

Дисперсия вещественной наблюдаемой $M$

$$
\mathbf{D}_{S}(M)=\int_{\mathscr{X}}\left(x-\mathbf{E}_{S}(M)\right)^{2} \mu_{S}^{M}(d x) .
$$

Если $M$ - проекторнозначная мера, то в предположении конечности второго момента

$$
\mathbf{D}_{S}(M) \equiv \mathbf{D}_{S}(A)=\operatorname{Tr} S\left(A-\mathbf{E}_{S}(M) I\right)^{2} .
$$

Если $S=|\psi\rangle\langle\psi|$ - чистое состояние, то конечность второго момента равносильна условию $\psi \in \mathscr{D}(A)$ (область определения оператора $A$ ) и

$$
\begin{aligned}
\mathbf{E}_{S}(A) & =\langle\psi \mid A \psi\rangle, \\
\mathbf{D}_{S}(A) & =\left\|\left(A-\mathbf{E}_{S}(M) I\right) \psi\right\|^{2} .
\end{aligned}
$$

3. Ковариантные оценки. Пусть $\mathscr{H}$ - гильбертово пространство наблюдаемой квантовой системы. Далее $G$ обозначает либо вещественную прямую $\mathbf{R}$ (в случае параметра смещения), либо одномерный тор $\mathbf{T}$ (в случае параметра поворота), и пусть $x \rightarrow V_{x}=e^{-i x A}, x \in G$, унитарное представление одномерной абелевой группы $G$ в $\mathscr{H}$. Спектр $\Lambda$ оператора $A$ является подмножеством двойственной группы $\widehat{G}$, которая совпадает с $\mathbf{R}$ в случае $G=\mathbf{R}$ и с группой целых чисел $\mathbf{Z}$, если $G=\mathbf{T}$. Мы рассматриваем задачу оценивания параметра сдвига $\theta \in G$ в семействе состояний

$$
S_{\theta}=e^{-i \theta A} S e^{i \theta A}
$$

где $S$ - начальное состояние, предполагаемое известным. Оиенкой является произвольная наблюдаемая со значениями в $G$.

Оценка $M$ называется ковариантной, если

$$
V_{x}^{*} M(B) V_{x}=M(B-x) \quad \text { для } B \subset G, x \in G,
$$

где $B-x:=\{y ; y+x \in B\}$ и в случае $G=\mathrm{T}$ имеется в виду сложение по модулю $2 \pi$. Необходимым и достаточным условием существования ковариантной оченки является абсолютная непрерывность спектра оператора $A$ относительно мерь Хара в двойственной группе $\widehat{G}$ [15], что мы и предположим. В случае $G=\mathbf{R}$ это означает, что $A$ имеет лебегов 
спектр, тогда как в случае $G=\mathrm{T}($ с $\widehat{G}=\mathrm{Z})$ это не налагает никаких ограничений. Введем операторы

$$
U_{y}:=\int_{G} e^{i y x} M(d x), \quad y \in \widehat{G} .
$$

Тогда (8) сводится к коммутационному соотношению Вейля

$$
U_{y} V_{x}=e^{i x y} V_{x} U_{y}, \quad x \in G, \quad y \in \widehat{G}
$$

(в котором, однако, оператор $U_{y}$, вообще говоря, не унитарен). В этом смысле наблюдаемая $M$ является канонически сопряженной к генератору группы сдвигов $A$.

Вводя характеристическую функцию

$$
\varphi_{S}^{M}(\lambda)=\mathbf{E}_{S}\left(U_{\lambda}\right)=\int_{G} e^{i \lambda x} \mu_{S}^{M}(d x), \quad \lambda \in \widehat{G},
$$

можно получить следующее соотношение неопределенностей для обобщенной канонической пары $(A, M)[14]$ :

$$
\Delta_{S}^{M}(\lambda) \cdot \mathbf{D}_{S}(A) \geqslant \frac{1}{4}
$$

где $\Delta_{S}^{M}(\lambda):=\lambda^{-2}\left(\left|\varphi_{S}^{M}(\lambda)\right|^{-2}-1\right), \lambda \neq 0$, служит функциональной мерой неопределенности ковариантной наблюдаемой $M$ в состоянии $S$. Если $G=\mathbf{R}$ и $M$ имеет конечную дисперсию $\mathbf{D}_{S}(M)$, то $\lim _{\lambda \rightarrow 0} \Delta_{S}^{M}(\lambda)=$ $\mathbf{D}_{S}(M)$, так что из (11) следует обобщение соотношения неопределенностей Гейзенберга

$$
\mathbf{D}_{S}(M) \cdot \mathbf{D}_{S}(A) \geqslant \frac{1}{4} \text {. }
$$

Основной интерес представляют ковариантные оценки с минимальной неопределенностью. В случае чистого начального состояния их описание дается следующей теоремой [15]. Пусть $S=|\psi\rangle\langle\psi|$, где $\psi-$ единичный вектор в $\mathscr{H}$, так что

$$
S_{\theta}=e^{-i \theta A}|\psi\rangle\langle\psi| e^{i \theta A}
$$

Поскольку спектр $A$ абсолютно непрерывен, имеет место спектральное разложение в форме прямого интеграла

$$
\mathscr{H}=\int_{\Lambda} \oplus \mathscr{H}(\lambda) d \lambda,
$$

диагонализующего унитарную группу $\left\{e^{i \theta A}\right\}$. Это означает, что для любого вектора

имеет место

$$
|\varphi\rangle=\int_{\Lambda} \oplus|\varphi(\lambda)\rangle d \lambda, \quad|\varphi(\lambda)\rangle \in \mathscr{H}(\lambda),
$$

$$
e^{i \theta A}|\varphi\rangle=\int_{\Lambda} \oplus e^{i \theta \lambda}|\varphi(\lambda)\rangle d \lambda, \quad \theta \in G
$$


Условимся о продолжении функции $\varphi(\lambda)$ на всю группу $\widehat{G}$, полагая ее равной нулю вне $\Lambda$.

Теорема 1 [14]. Для любой ковариантной оченки $M$

$$
\begin{aligned}
\left|\varphi_{S}^{M}(\lambda)\right| \leqslant \varphi_{S}^{*}(\lambda) & :=\int_{\widehat{G}}\left\|\psi\left(\lambda^{\prime}\right)\right\|\left\|\psi\left(\lambda^{\prime}+\lambda\right)\right\| d \lambda^{\prime}, \\
\Delta_{S}^{M}(\lambda) \geqslant \Delta_{S}^{*}(\lambda): & =\lambda^{-2}\left(\varphi_{S}^{*}(\lambda)^{-2}-1\right) .
\end{aligned}
$$

$B$ cлyчue $G=\mathbf{R}$

$$
\mathbf{D}_{S}(M) \geqslant \mathbf{D}_{S}^{*}:=\int_{\mathbf{R}}\left(\frac{d}{d \lambda}\|\psi(\lambda)\|\right)^{2} d \lambda
$$

при условии, что правая часть определена и конечна.

Равенства достигаются на оптимальной ковариантной наблюдаемой $M_{*}$, которая задается следующим ядром в интегральном разложении (13):

$$
M_{*}(d x)=\left[e^{i x\left(\lambda^{\prime}-\lambda\right)} \frac{|\psi(\lambda)\rangle\left\langle\psi\left(\lambda^{\prime}\right)\right|}{\|\psi(\lambda)\|\left\|\psi\left(\lambda^{\prime}\right)\right\|}\right] \frac{d x}{2 \pi}
$$

Отметим, что оператор

$$
P_{*}:=\int_{G} M_{*}(d x)=\left[\delta\left(\lambda^{\prime}-\lambda\right) \frac{|\psi(\lambda)\rangle\left\langle\psi\left(\lambda^{\prime}\right)\right|}{\|\psi(\lambda)\|\left\|\psi\left(\lambda^{\prime}\right)\right\|}\right]
$$

является проектором на инвариантное подпространство группы $\left\{e^{i \theta A}\right\}$, порожденное векторами $e^{i \theta A}|\psi\rangle, \theta \in G$. В самом деле, $P_{*} e^{i \theta A}|\psi\rangle=$ $e^{i \theta A}|\psi\rangle$, и если вектор $|\varphi\rangle$ ортогонален всем этим векторам, то $\int_{\Lambda} e^{i \lambda \theta}\langle\varphi(\lambda) \mid \psi(\lambda)\rangle d \lambda=0, \theta \in G$, и $\langle\varphi(\lambda) \mid \psi(\lambda)\rangle=0$ для $\lambda \in \Lambda$, откуда $P_{*}|\varphi\rangle=0$. Таким образом, мера $M_{*}$ в общем случае лишь субнормирована, и ее следует доопределить на ортогональном дополнении подпространства $\mathscr{H}_{*}=P_{*} \mathscr{H}$, чтобы получить наблюдаемую. Независимо от способа продолжения она имеет следуюшую плотность распределения вероятностей в состоянии $S_{\theta}$, являюшуюся преобразованием Фурье характеристической функции (14):

$$
p_{\theta}^{*}(x)=\frac{1}{2 \pi}\left|\int e^{i \lambda(x-\theta)}\|\psi(\lambda)\| d \lambda\right|^{2}
$$

В [15] показано также, что $M_{*}$ минимизирует среднее отклонение $\mathscr{R}(M)=\int W(x-\theta) p_{\theta}^{M}(x) d x$, где $W-$ произвольная непрерьвная условно отрицательно определенная функиия.

Аналогично (18), получаем формулу

$$
U_{* y}:=\int_{G} e^{i y x} M_{*}(d x)=\left[\delta\left(y+\lambda^{\prime}-\lambda\right) \frac{|\psi(\lambda)\rangle\left\langle\psi\left(\lambda^{\prime}\right)\right|}{\|\psi(\lambda)\|\left\|\psi\left(\lambda^{\prime}\right)\right\|}\right],
$$

которая будет использована в дальнейшем. 
П р и м е р (см. [1], [2]). Рассмотрим случай $\mathscr{H}=L^{2}(\mathbf{R})$, где $A$ действует как оператор умножения на независимую переменную $\lambda$ (импульсное представление). Тогда $\theta$ является параметром смешения положения. В этом случае $\mathscr{H}(\lambda) \simeq \mathbf{R},\|\psi(\lambda)\|=|\psi(\lambda)|$ и (17) является ортогональным разложением единицы в $\mathscr{H}$. Для доказательства проще всего проверить, что операторы (19) образуют унитарную группу. Это разложение единицы является спектральной мерой самосопряженного опеparopa

$$
Q_{*}=\int x M_{*}(d x)=\left.\frac{1}{i} \frac{d}{d y}\right|_{y=0} U_{* y} .
$$

Из (19) вытекает, что действие этого оператора на вектор $\varphi$ дается выражением

$$
Q_{*} \varphi(\lambda)=e^{i \alpha(\lambda)} Q e^{-i \alpha(\lambda)} \varphi(\lambda),
$$

где $e^{i \alpha(\lambda)}=\psi(\lambda) /\|\psi(\lambda)\|, Q=i \frac{d}{d \lambda}-$ оператор положения в импульсном представлении. Таким образом, оптимальная ковариантная наблюдаемая с точностью до калибровочного преобразования, компенсирующего фазу $\alpha(\lambda)$ вектора состояния $|\psi\rangle$ в импульсном представлении, совпадает с наблюдаемой положения. Если фаза $\alpha(\lambda)$ абсолютно непрерывна, то

$$
Q_{*} \varphi(\lambda)=\left[Q+\alpha^{\prime}(\lambda) I\right] \varphi(\lambda)
$$

для функций $\varphi(\lambda)$ из подходящей области определения.

4. Многократные наблюдения. Теперь мы рассмотрим задачу оценивания параметра сдвига $\theta \in \mathbf{R}$ в семействе состояний

$$
S_{\theta}^{\otimes n}=S_{\theta} \otimes \cdots \otimes S_{\theta}
$$

в гильбертовом пространстве $\mathscr{H}^{\otimes n}=\mathscr{H} \otimes \cdots \otimes \mathscr{H}(n$-кратное тензорное произведение, которое соответствует $n$ независимым наблюдениям). Здесь $S_{\theta}=e^{-i \theta A} S e^{i \theta A}$, где $S=|\psi\rangle\langle\psi|$ - чистое состояние.

Семейство (21) ковариантно относительно унитарного представления группы сдвигов $\mathbf{R}$

$$
\theta \rightarrow \exp \left(-i \theta A^{(n)}\right), \quad A^{(n)}=A \otimes \cdots \otimes I+\cdots+I \otimes \cdots \otimes A
$$

в $\mathscr{H}^{\otimes n}$. Соответствующее разложение в прямой интеграл имеет вид

$$
\mathscr{H}^{\otimes n}=\int \oplus \mathscr{H}^{(n)}(\lambda) d \lambda,
$$

где

$$
\begin{aligned}
\mathscr{H}^{(n)}(\lambda)=\int \oplus[ & \mathscr{H}\left(\lambda_{1}\right) \otimes \cdots \otimes \mathscr{H}\left(\lambda_{n-1}\right) \\
& \left.\otimes \mathscr{H}\left(\lambda-\lambda_{1}-\cdots-\lambda_{n-1}\right)\right] d \lambda_{1} \cdots d \lambda_{n-1}
\end{aligned}
$$


Для симметрии обозначений мы будем также записывать это как

$$
\int_{\Gamma(\lambda)} \oplus\left[\mathscr{H}\left(\lambda_{1}\right) \otimes \cdots \otimes \mathscr{H}\left(\lambda_{n}\right)\right] d^{n-1} \sigma
$$

где

$$
\Gamma(\lambda)=\left\{\left(\lambda_{1}, \ldots, \lambda_{n}\right): \lambda_{1}+\cdots+\lambda_{n}=\lambda\right\} .
$$

Тогда $\left|\psi^{\otimes n}\right\rangle=\int \oplus\left|\psi^{(n)}(\lambda)\right\rangle d \lambda$, где

$$
\left|\psi^{(n)}(\lambda)\right\rangle=\int_{\Gamma(\lambda)} \oplus\left[\left|\psi\left(\lambda_{1}\right)\right\rangle \otimes \cdots \otimes\left|\psi\left(\lambda_{n}\right)\right\rangle\right] d^{n-1} \sigma,
$$

так что

$$
\left\|\psi^{(n)}(\lambda)\right\|^{2}=\int_{\Gamma(\lambda)} \oplus\left[\left\|\psi\left(\lambda_{1}\right)\right\|^{2} \otimes \cdots \otimes\left\|\psi\left(\lambda_{n}\right)\right\|^{2}\right] d^{n-1} \sigma .
$$

Используя эти обозначения, оптимальную ковариантную оценку можно записать в виде (17):

$$
M_{*}^{(n)}(d x)=\left[e^{i x\left(\lambda^{\prime}-\lambda\right)} \frac{\left|\psi^{(n)}(\lambda)\right\rangle\left\langle\psi^{(n)}\left(\lambda^{\prime}\right)\right|}{\left\|\psi^{(n)}(\lambda)\right\|\left\|\psi^{(n)}\left(\lambda^{\prime}\right)\right\|}\right] \frac{d x}{2 \pi} .
$$

Однако в этом случае проектор

$$
P_{*}^{(n)}:=\int M_{*}^{(n)}(d x)=\left[\delta\left(\lambda^{\prime}-\lambda\right) \frac{\left|\psi^{(n)}(\lambda)\right\rangle\left\langle\psi^{(n)}\left(\lambda^{\prime}\right)\right|}{\left\|\psi^{(n)}(\lambda)\right\|\left\|\psi^{(n)}\left(\lambda^{\prime}\right)\right\|}\right], \quad n>1,
$$

не может равняться единичному оператору: во всяком случае, он проецирует на подпространство $\mathscr{H}_{*}^{(n)}$, лежащее в подпространстве векторфункций, симметрично зависяших от $\lambda_{1}, \ldots, \lambda_{n}$.

Обозначим $p(\lambda)=\|\psi(\lambda)\|^{2}$ и $p^{(n)}(\lambda)=\left\|\psi^{(n)}(\lambda)\right\|^{2}$ плотности распределения вероятностей наблюдаемых $A$ и $A^{(n)}$ в состояниях $S$ и $S^{\otimes n}$ соответственно. Тогда из (22) вытекает, что $p^{(n)}(\lambda)$ есть $n$-кратная свертка $p(\lambda)^{* n}$. Мы предполагаем, что $p(\lambda)$ и, следовательно, $p^{(n)}(\lambda)-$ дифференцируемые функции. Особенно полезным в настоящем контексте является понятие слабой дифференцируемости [13]: плотность распределения вероятностей $p(\lambda)$ называется слабо дифференцируемой, если найдется функция $s(\cdot) \in L^{2}(p)$ такая, что для всех $f$ с $\int|f(\lambda)|^{2} p(\lambda+$ $\theta) d \lambda<\infty$ функция $g(\theta)=\int f(\lambda) p(\lambda+\theta) d \lambda$ имеет. производную $g^{\prime}(\theta)=$ $\int f(\lambda) s(\lambda+\theta) p(\lambda+\theta) d \lambda$.

Чтобы лучше понять структуру оптимальной ковариантной оценки при $n>1$, рассмотрим ситуацию примера. Тогда $\mathscr{H}^{\otimes n}$ изоморфно $L^{2}\left(\mathbf{R}^{n}\right)$ и $A^{(n)}$ является просто оператором умножения на $\lambda=\lambda_{1}+\cdots+\lambda_{n}$.

Теорема 2. Оптимальная ковариантная наблюдаемая (23) является спектральной мерой самосопряженного оператора

$$
Q_{*}^{(n)}=P_{*}^{(n)} \frac{1}{n}\left(Q_{*} \otimes \cdots \otimes I+\cdots+I \otimes \cdots \otimes Q_{*}\right) P_{*}^{(n)} .
$$


Наблюдаемая $n^{-1}\left(Q_{*} \otimes \cdots \otimes I+\cdots+I \otimes \cdots \otimes Q_{*}\right)$ отвечает «полуклассическому» методу оценивания, при котором сначала находятся оптимальные квантовые оценки для каждой из $n$ компонент в $\mathscr{H}^{\otimes n}$, результаты которых потом используются классически для вычисления среднего по $n$ наблюдениям. Теорема показывает, что проецируя это среднее на $\mathscr{H}_{*}^{(n)}$ (и таким образом вводя сцепленность), мы получаем оптимальную ковариантную оценку по $n$ наблюдениям.

Д ок азат е л ь с т о. Аналогично рассмотренному примеру, можно показать, что (23) является ортогональным разложением единицы. Как следует из $(24)$, подпространство $\mathscr{H}_{*}^{(n)}=P_{*}^{(n)} \mathscr{H}$ состоит из функций вида

$$
\varphi\left(\lambda_{1}, \ldots, \lambda_{n}\right)=\frac{c(\lambda)}{\sqrt{p^{(n)}(\lambda)}} \psi\left(\lambda_{1}\right) \cdots \psi\left(\lambda_{n}\right),
$$

где $\int|c(\lambda)|^{2} d \lambda=\|\varphi\|^{2}$. Рассмотрим действие на эти функции самосопряженного оператора

$$
Q_{*}^{(n)}=\int x M_{*}^{(n)}(d x)=\left[\int x \frac{e^{-i x \lambda}\left|\psi^{(n)}(\lambda)\right\rangle\left\langle\psi^{(n)}\left(\lambda^{\prime}\right)\right| e^{i x \lambda^{\prime}}}{\left\|\psi^{(n)}(\lambda)\right\|\left\|\psi^{(n)}\left(\lambda^{\prime}\right)\right\|} \frac{d x}{2 \pi}\right] .
$$

(Отметим формальную аналогию между этим выражением и формулой Питмена

$$
\theta_{*}=\frac{\int \theta \tilde{p}\left(x_{1}-\theta\right) \cdots \widetilde{p}\left(x_{n}-\theta\right) d \theta}{\int \widetilde{p}\left(x_{1}-\theta\right) \cdots \widetilde{p}\left(x_{n}-\theta\right) d \theta}
$$

для классической оптимальной эквивариантной оценки параметра сдвига $\theta$ в семействе $\left\{\tilde{p}\left(x_{1}-\theta\right) \cdots \tilde{p}\left(x_{n}-\theta\right)\right\}$, см., например, [10].) Используя аналог соотношения (20), можно показать, что для $\varphi \in \mathscr{H}_{*}^{(n)}$

$$
Q_{*}^{(n)} \varphi\left(\lambda_{1}, \ldots, \lambda_{n}\right)=i \frac{c^{\prime}(\lambda)}{\sqrt{p^{(n)}(\lambda)}} \psi\left(\lambda_{1}\right) \cdots \psi\left(\lambda_{n}\right)
$$

при условии, что $c(\lambda)$ абсолютно непрерывна, а $c^{\prime}(\lambda)$ квадратично интегрируема. Имеем

$$
c^{\prime}(\lambda)=\frac{1}{n} \sum_{j=1}^{n} \frac{\partial}{\partial \lambda_{j}} c\left(\lambda_{1}+\cdots+\lambda_{n}\right)
$$

откуда

$$
\begin{gathered}
Q_{*}^{(n)} \varphi\left(\lambda_{1}, \ldots, \lambda_{n}\right)=\frac{i \sum_{j=1}^{n} \partial / \partial \lambda_{j}}{n}\left[\frac{c\left(\lambda_{1}+\cdots+\lambda_{n}\right)}{\sqrt{p^{(n)}\left(\lambda_{1}+\cdots+\lambda_{n}\right)}} \psi\left(\lambda_{1}\right) \cdots \psi\left(\lambda_{n}\right)\right] \\
-c\left(\lambda_{1}+\cdots+\lambda_{n}\right) \frac{i \sum_{j=1}^{n} \partial / \partial \lambda_{j}}{n}\left[\frac{\psi\left(\lambda_{1}\right) \cdots \psi\left(\lambda_{n}\right)}{\sqrt{p^{(n)}\left(\lambda_{1}+\cdots+\lambda_{n}\right)}}\right] .
\end{gathered}
$$


Принимая во внимание, что $\psi\left(\lambda_{j}\right)=\sqrt{p\left(\lambda_{j}\right)} e^{i \alpha\left(\lambda_{j}\right)}$, и выполняя дифференцирование, мы получаем после некоторых преобразований

$$
\begin{aligned}
Q_{*}^{(n)} \varphi\left(\lambda_{1}, \ldots, \lambda_{n}\right)= & \frac{\sum_{j=1}^{n}\left(i \partial / \partial \lambda_{j}+\alpha^{\prime}\left(\lambda_{j}\right)\right)}{n} \varphi\left(\lambda_{1}, \ldots, \lambda_{n}\right) \\
& +\frac{i}{2} F\left(\lambda_{1}, \ldots, \lambda_{n}\right) \varphi\left(\lambda_{1}, \ldots, \lambda_{n}\right),
\end{aligned}
$$

где

$$
F\left(\lambda_{1}, \ldots, \lambda_{n}\right)=\frac{\left[p^{(n)}(\lambda)\right]^{\prime}}{p^{(n)}(\lambda)}-\frac{1}{n} \sum_{j=1}^{n} \frac{p^{\prime}\left(\lambda_{j}\right)}{p\left(\lambda_{j}\right)}
$$

- вешественная функция, или, кратко,

$$
Q_{*}^{(n)} \varphi=\frac{1}{n}\left(Q_{*} \otimes \cdots \otimes I+\cdots+I \otimes \cdots \otimes Q_{*}\right) \varphi+\frac{i}{2} F \varphi, \quad \varphi \in \mathscr{H}_{*}^{(n)} .
$$

Вычисляя скалярное произведение с $\varphi$ и замечая, что как $Q_{*}^{(n)}$, так и

$$
\frac{1}{n}\left(Q_{*} \otimes \cdots \otimes I+\cdots+I \otimes \cdots \otimes Q_{*}\right)
$$

- самосопряженные операторы, имеем $\langle\varphi \mid F \varphi\rangle=0, \varphi \in \mathscr{H}_{*}^{(n)}$, откуда следует (25).

Можно также дать прямое доказательство того, что $\langle\varphi \mid F \varphi\rangle=0$, если $\varphi$ дается соотношением (26). Имеем

$$
\begin{aligned}
\langle\varphi \mid F \varphi\rangle=\int\left\{\frac{\left[p^{(n)}(\lambda)\right]^{\prime}}{p^{(n)}(\lambda)}-\frac{1}{n} \sum_{j=1}^{n} \frac{p^{\prime}\left(\lambda_{j}\right)}{p\left(\lambda_{j}\right)}\right\} \frac{|c(\lambda)|^{2}}{p^{(n)}(\lambda)} p\left(\lambda_{1}\right) \cdots p\left(\lambda_{n}\right) d \lambda_{1} \cdots d \lambda_{n} \\
=\int d \lambda \frac{|c(\lambda)|^{2}}{p^{(n)}(\lambda)} \int_{\Gamma(\lambda)}\left\{\frac{\left[p^{(n)}(\lambda)\right]^{\prime} p\left(\lambda_{1}\right) \cdots p\left(\lambda_{n}\right)}{p^{(n)}(\lambda)}\right. \\
\left.-\frac{1}{n} \sum_{j=1}^{n} \frac{\partial}{\partial \lambda_{j}} p\left(\lambda_{1}\right) \cdots p\left(\lambda_{n}\right)\right\} d^{n-1} \sigma .
\end{aligned}
$$

Ho

$$
\begin{aligned}
p^{(n)}(\lambda) & =\int_{\Gamma(\lambda)} p\left(\lambda_{1}\right) \cdots p\left(\lambda_{n}\right) d^{n-1} \sigma=p(\lambda)^{* n} \\
& =\int p\left(\lambda_{1}\right) \cdots p\left(\lambda_{n-1}\right) p\left(\lambda-\lambda_{1}-\cdots-\lambda_{n-1}\right) d \lambda_{1} \cdots d \lambda_{n-1} .
\end{aligned}
$$

Дифференцируя последнее тождество по $\lambda$, мы получаем

$$
\begin{aligned}
{\left[p^{(n)}(\lambda)\right]^{\prime} } & =\int p\left(\lambda_{1}\right) \cdots p\left(\lambda_{n-1}\right) p^{\prime}\left(\lambda-\lambda_{1}-\cdots-\lambda_{n-1}\right) d \lambda_{1} \cdots d \lambda_{n-1} \\
& =\int_{\Gamma(\lambda)} \frac{\partial}{\partial \lambda_{n}} p\left(\lambda_{1}\right) \cdots p\left(\lambda_{n}\right) d^{n-1} \sigma
\end{aligned}
$$

и аналогично для всех $\lambda_{j}$. Поэтому

$$
\left[p^{(n)}(\lambda)\right]^{\prime}=\frac{1}{n} \int_{\Gamma(\lambda)} \sum_{j=1}^{n} \frac{\partial}{\partial \lambda_{j}} p\left(\lambda_{1}\right) \cdots p\left(\lambda_{n}\right) d^{n-1} \sigma .
$$


Принимая во внимание (29), получаем из (30), что внутренний интеграл в (28) равен нулю.

5. Предельная теорема. В этом пункте мы делаем следующее предположение регулярности.

(A) $\psi \in \mathscr{D}(A)$, или, что равносильно, $\|A \psi\|^{2}=\int \lambda^{2} p(\lambda) d \lambda<\infty$, где $p(\lambda)=\|\psi(\lambda)\|^{2}$ - плотность распределения вероятностей наблюдаемой $A$ в состоянии $S$. Без ограничения общности предположим, ито $\mathbf{E}_{\psi}(A)=\langle\psi \mid A \psi\rangle=\int \lambda p(\lambda) d \lambda=0$, mогда $\mathbf{D}_{S}(A)=\int \lambda^{2} p(\lambda) d \lambda$ дисперсия $A$.

Соотношение неопределенностей (12) вместе с (16) влекут нижнюю границу для дисперсии оптимальной ковариантной оценки

$$
\mathrm{D}_{S^{(n)}}^{*}=\int_{\mathbf{R}}\left(\frac{d}{d \lambda} \sqrt{p(\lambda)^{* n}}\right)^{2} d \lambda \geqslant \frac{1}{4 \mathbf{D}_{S^{(n)}}\left(A^{(n)}\right)}=\frac{1}{4 n \mathbf{D}_{S}(A)},
$$

где равенство достигается в том и только том случае, когда $p(\lambda)$ гауссовская плотность. Величина $\mathbf{D}_{S_{(n)}^{*}}$ представляет собой достижимую нижнюю границу для дисперсий произвольных (в общем случае сцепленных) ковариантных оценок параметра сдвига, использующих $n$ независимых наблюдений.

Согласно локальной центральной предельной теореме в $L^{1}[16]$,

$$
p_{n}(\lambda):=\sqrt{n} \sigma p(\sqrt{n} \sigma \lambda)^{* n} \longrightarrow p_{0}(\lambda):=\frac{1}{\sqrt{2 \pi}} e^{-\lambda^{2} / 2} .
$$

Следовательно,

$$
\int\left|\sqrt{p_{0}(\lambda)}-\sqrt{p_{n}(\lambda)}\right|^{2} d \lambda \leqslant \int\left|p_{0}(\lambda)-p_{n}(\lambda)\right| d \lambda \longrightarrow 0 .
$$

Поэтому преобразование Фурье функции $\sqrt{p_{n}(\lambda)}$ сходится к преобразованию Фурье функции $\sqrt{p_{0}(\lambda)}$ в $L^{2}$, и плотность распределения вероятностей оптимальной ковариантной наблюдаемой

$$
p_{S^{(n)}}^{*}(x)=\frac{1}{2 \pi}\left|\int e^{-i x \lambda} \sqrt{p(\lambda)^{* n}} d \lambda\right|^{2}
$$

удовлетворяет локальной предельной теореме

$$
\int\left|\sqrt{\frac{2}{\pi}} e^{-2 x^{2}}-\frac{1}{\sqrt{n} \sigma} p_{S^{(n)}}^{*}\left(\frac{x}{\sqrt{n} \sigma}\right)\right| d x \longrightarrow 0 .
$$

Таким образом, в предположении (А) распределение вероятностей оптимальной ковариантной наблюдаемой $M_{*}^{(n)}$ в состоянии $S_{\theta}$ асимптотически нормально с параметрами $\left(\theta,\left(4 n \sigma^{2}\right)^{-1}\right)$. В этом смысле граница соотношения неопределенностей достигается асимптотически. Более того, используя основной результат работы [13], получаем асимптотическую эффективность. 
Теорема 3. Если плотность $p(\lambda)$ слабо дифферениируема и дисперсия $\mathbf{D}_{S_{(n)}^{*}}^{*}$ конечна для некоторого $n$, то

$$
\lim _{n \rightarrow \infty} n \mathbf{D}_{S^{(n)}}^{*}=\frac{1}{4 \mathbf{D}_{S}(A)}
$$

6. Полуклассическое оценивание. Рассмотрим теперь способ оценивания, при котором оптимальная ковариантная квантовая оценка $M_{*}^{(1)}(d x)$ находится для каждой из $n$ компонент тензорного произведения $\mathscr{H}^{\otimes n}$, а затем производится классическое оценивание по полученным исходам $n$ наблюдений. Плотность распределения вероятностей наблюдаемой $M_{*}^{(1)}(d x)$ в состоянии $S_{\theta}$ равна

$$
\widetilde{p}_{\theta}(x)=\frac{1}{2 \pi}|\psi(x-\theta)|^{2},
$$

где $\psi(x)=\int e^{i \lambda x}\|\psi(\lambda)\| d \lambda$. В предположении (А) $\psi(x)$, а следовательно, и $\tilde{p}_{\theta}(x)$ дифференцируемы, и для любой несмещенной оценки по $n$ наблюдениям имеет место классическое неравенство Рао-Крамера:

$$
\mathbf{D}_{n} \geqslant\left[n \int \frac{\left|\tilde{p}_{\theta}^{\prime}(x)\right|^{2}}{\widetilde{p}_{\theta}(x)} d x\right]^{-1} .
$$

Заметим, что

$$
\int \frac{\left|\widetilde{p}_{\theta}^{\prime}(x)\right|^{2}}{\widetilde{p}_{\theta}(x)} d x=4 \int \frac{\left|\operatorname{Re} \bar{\psi}^{\prime}(x) \psi(x)\right|^{2}}{|\psi(x)|^{2}} \frac{d x}{2 \pi}=4 \int\left[|\psi(x)|^{\prime}\right]^{2} \frac{d x}{2 \pi} .
$$

С другой стороны,

$$
\int \lambda^{2}\|\psi(\lambda)\|^{2} d \lambda=\int\left|\psi^{\prime}(x)\right|^{2} \frac{d x}{2 \pi} .
$$

Сравнивая это с асимптотически достижимой квантовой границей соотношения неопределенностей, имеем

$$
\int\left[|\psi(x)|^{\prime}\right]^{2} \frac{d x}{2 \pi}<\int\left|\psi^{\prime}(x)\right|^{2} \frac{d x}{2 \pi}=\int\left[|\psi(x)|^{\prime}\right]^{2} \frac{d x}{2 \pi}+\int\left|\beta^{\prime}(x) \psi(x)\right|^{2} \frac{d x}{2 \pi}
$$

если только $\beta(x):=\arg \psi(x) \neq$ const. Таким образом, при этом условии для достаточно больших $n$

$$
n \min \mathbf{D}_{n}>n \mathbf{D}_{S^{(n)}}^{*},
$$

где минимум берется по всем несмещенным классическим оценкам, использующим несцепленные ковариантные квантовые наблюдаемые.

7. Нерегулярный случай. Рассмотрим теперь в ситуации примера случай, когда условие регулярности (А) не выполняется. Пусть $S=|\psi\rangle\langle\psi| \mathrm{c}$

$$
\psi(\lambda)=\frac{1}{\sqrt{\pi a}} \frac{\sin a \lambda}{\lambda}
$$


так что $\mathbf{D}_{S}(A)=\infty$. В координатном представлении это отвечает прямоугольной функции

$$
\widetilde{\psi}(x)=\int e^{-i \lambda x} \psi(\lambda) d \lambda=\left\{\begin{array}{lll}
\sqrt{\frac{\pi}{a}}, & \text { если } & x \in[-a, a] ; \\
0, & \text { если } & x \notin[-a, a],
\end{array}\right.
$$

т.е. равномерному распределению положения частицы в отрезке $[-a, a]$, с плотностью $\tilde{p}(x)=(2 \pi)^{-1}|\widetilde{\psi}(x)|^{2}$ в $\mathbf{R} . \mathrm{K}$ сожалению, плотность распределения вероятностей оптимальной ковариантной наблюдаемой $Q_{*}$, равная

$$
p^{*}(x)=\frac{1}{2 \pi}\left|\int e^{-i \lambda x}\right| \psi(\lambda)|d \lambda|^{2},
$$

не может быть найдена в явном виде, поэтому мы рассмотрим полуклассическое оценивание, основанное на немодифицированной наблюдаемой положения $Q$, имеющей плотность распределения вероятностей $\widetilde{p}(x-\theta)$.

Обращаясь к случаю $n$ наблюдений, обозначим

$$
Q_{1}=Q \otimes \cdots \otimes I, \ldots, Q_{n}=I \otimes \cdots \otimes Q .
$$

Как хорошо известно (см., например, [17, п. 28.6]), оценка Питмена (27) в случае прямоугольной плотности вероятностей $\widetilde{p}(x)$ имеет вид

$$
\theta_{*}=\frac{1}{2}\left[\min \left(Q_{1}, \ldots, Q_{n}\right)+\max \left(Q_{1}, \ldots, Q_{n}\right)\right] .
$$

Ее дисперсия равна

$$
\mathrm{D}_{S}\left(\theta_{*}\right)=\frac{2 a^{2}}{(n+1)(n+2)} \sim \frac{2 a^{2}}{n^{2}} .
$$

Прямоугольная плотность недифференцируема, что нарушает условие регулярности и делает неприменимым неравенство Рао-Крамера. Далее, обозначая $p_{n}(x)$ плотность распределения оценки $\theta_{*}$, имеем предельное распределение [17]

$$
\lim _{n \rightarrow \infty} n^{-1} p_{n}\left(\frac{x}{n}\right)=\frac{1}{2 a} e^{-|x| / a} .
$$

Найдем теперь асимптотику оптимальной квантовой ковариантной наблюдаемой (25). Обозначая

$$
p(\lambda)=|\psi(\lambda)|^{2}=\frac{1}{\pi a}\left(\frac{\sin a \lambda}{\lambda}\right)^{2}
$$

плотность распределения вероятностей наблюдаемой $A$ и $f(x)=$ $\int e^{-i \lambda x} p(\lambda) d \lambda$, имеем

$$
f(x)=\left\{\begin{array}{lll}
1-\frac{|x|}{2 a}, & \text { если } & x \in[-2 a, 2 a] ; \\
0, & \text { если } & x \notin[-a, a] .
\end{array}\right.
$$


Таким образом,

откуда

$$
p(\lambda)^{* n}=\frac{1}{2 \pi} \int e^{i \lambda x} f(x)^{n} d x
$$

$$
\begin{aligned}
\lim _{n \rightarrow \infty} n p_{n}(n \lambda)^{* n} & =\frac{1}{2 \pi} \lim _{n \rightarrow \infty} \int e^{i \lambda x} f\left(\frac{x}{n}\right)^{n} d x \\
& =\frac{1}{2 \pi} \int \exp \left(i \lambda x-\frac{|x|}{2 a}\right) d x=\frac{1}{2 \pi a} \frac{1}{\lambda^{2}+(2 a)^{-2}} .
\end{aligned}
$$

Принимая во внимание, что

$$
\int e^{-i \lambda x} \frac{1}{\sqrt{\lambda^{2}+(2 a)^{-2}}} d \lambda=2 K_{0}\left(\frac{|x|}{2 a}\right)
$$

где $K_{0}$ - функция Макдональда (модифицированная функция Бесселя), и используя предельное распределение, можно показать (см. приложение), что плотность распределения вероятностей оптимальной ковариантной наблюдаемой

$$
p_{S^{(n)}}^{*}(x)=\frac{1}{2 \pi}\left|\int e^{-i \lambda x} \sqrt{p(\lambda)^{* n}} d \lambda\right|^{2}
$$

удовлетворяет предельному соотношению

$$
\left.\lim _{n \rightarrow \infty} \int\left|\frac{1}{n} p_{S^{(n)}}^{*}\left(\frac{x}{n}\right)-\frac{2}{\pi a}\right| K_{0}\left(\frac{|x|}{2 a}\right)\right|^{2} \mid d x=0 .
$$

Дополнительное рассуждение (см. приложение) показывает, что ее дисперсия имеет асимптотику

$$
\begin{aligned}
\lim _{n \rightarrow \infty} n^{2} \mathbf{D}_{S^{(n)}}^{*} & =\lim _{n \rightarrow \infty} \int_{\mathbf{R}}\left(\frac{d}{d \lambda} \sqrt{n p(n \lambda)^{* n}}\right)^{2} d \lambda \\
& =\frac{1}{2 \pi a} \int_{\mathbf{R}}\left(\frac{d}{d \lambda} \sqrt{\frac{1}{\lambda^{2}+(2 a)^{-2}}}\right)^{2} d \lambda=\frac{a^{2}}{2},
\end{aligned}
$$

что в четыре раза меньше, чем соответствуюшее значение для полуклассической оценки $\theta_{*}$. Далее, $K_{0}(x) \sim \sqrt{\pi /(2 x)} e^{-x}$ для больших положительных $x$, откуда

$$
\frac{2}{\pi a}\left|K_{0}\left(\frac{|x|}{2 a}\right)\right|^{2} \sim \frac{2}{|x|} e^{-|x| / a}
$$

т.е. хвосты асимптотического распределения оптимальной оценки убывают быстрее, чем (31).

\section{8. Приложение.}

Доказательство соотношения (33). Обозначая

$$
\begin{aligned}
f_{0}(x) & =e^{-|x| /(2 a)}, & p_{0}(\lambda) & =\frac{1}{2 \pi a} \frac{1}{\lambda^{2}+(2 a)^{-2}}, \\
\Delta f_{n}(x) & =f_{0}(x)-f\left(\frac{x}{n}\right)^{n}, & \Delta p_{n}(\lambda) & =p_{0}(\lambda)-n p(n \lambda)^{* n}
\end{aligned}
$$


имеем

$$
\Delta p_{n}(\lambda)=\frac{1}{2 \pi} \int e^{i \lambda x} \Delta f_{n}(x) d x
$$

Теперь заметим, что

$$
\lim _{n \rightarrow \infty} \int_{x \neq 0}\left|\Delta f_{n}(x)^{(k)}\right||x|^{l} d x=0, \quad k, l=0,1,2, \ldots .
$$

Из (34), в частности, следует, что

$$
\max _{\lambda}\left|\Delta p_{n}(\lambda)\right| \leqslant \frac{1}{2 \pi} \int\left|\Delta f_{n}(x)\right| d x \longrightarrow 0 \quad \text { при } \quad n \rightarrow \infty .
$$

Оценим также хвосты распределения $\Delta p_{n}(\lambda)$. Принимая во внимание, что

$$
\Delta f_{n}(0)=\left(\Delta f_{n}(0)\right)^{\prime}=0,
$$

и дважды интегрируя по частям в (34), получаем

$$
\left|\Delta p_{n}(\lambda)\right| \leqslant \frac{1}{\lambda^{2}} \int_{x \neq 0}\left|\Delta f_{n}(x)^{\prime \prime}\right| d x=\frac{\varepsilon_{n}}{\lambda^{2}}
$$

где $\lim _{n \rightarrow \infty} \varepsilon_{n}=0$. Аналогично получаем

$$
\max _{\lambda}\left|\frac{d}{d \lambda} \Delta p_{n}(\lambda)\right| \leqslant \frac{1}{2 \pi} \int\left|x \Delta f_{n}(x)\right| d x \rightarrow 0 \quad \text { при } \quad n \rightarrow \infty
$$

и

$$
\left|\frac{d}{d \lambda} \Delta p_{n}(\lambda)\right| \leqslant \frac{1}{|\lambda|^{3}} \int_{x \neq 0}\left|\left(x \Delta f_{n}(x)\right)^{\prime \prime \prime}\right| d x=\frac{\varepsilon_{n}^{\prime}}{|\lambda|^{3}} .
$$

Из (35), (36) следует, что $\int\left|\Delta p_{n}(\lambda)\right| d \lambda \rightarrow 0$ при $n \rightarrow \infty$, откуда, рассуждая как в п. 4 , получаем $\sqrt{n p(n \lambda)^{* n}} \rightarrow \sqrt{p_{0}(\lambda)}$ в $L^{2}$, что влечет (32).

Оценки (35)-(38) вместе с

$$
p_{0}(\lambda) \asymp \frac{c}{1+\lambda^{2}}, \quad\left|\frac{d}{d \lambda} p_{0}(\lambda)\right| \asymp \frac{c}{1+|\lambda|^{3}}
$$

влекут

$$
\left|\left(\frac{d}{d \lambda} \sqrt{p_{0}(\lambda)}\right)^{2}-\left(\frac{d}{d \lambda} \sqrt{p_{n}(\lambda)}\right)^{2}\right|=\frac{1}{4}\left|\frac{p_{0}^{\prime}(\lambda)^{2}}{p_{0}(\lambda)}-\frac{p_{n}^{\prime}(\lambda)^{2}}{p_{n}(\lambda)}\right| \leqslant \frac{\varepsilon_{n}^{\prime \prime}}{1+\lambda^{2}}
$$

откуда следует (33).

9. Заключение и благодарности. В настоящей работе мы продемонстрировали преимущество, обусловленное квантовым свойством сцепленности, ограничиваясь классом ковариантных оценок. Следует отметить, что преимушество сцепленности проявляется и в более широком классе локально-несмещенных оценок, однако лишь в задачах оценивания многомерного параметра смешанного состояния [3]. Более подробно об асимптотической теории локально-несмещенных оценок параметров квантового состояния см. в работах [6]-[8]. Автор благодарит 
П. Харремоеса, который указал на работу [13], а также других участников и организаторов конференции «Квантовые измерения и квантовая стохастика», прошедшей в MaPhySto (Centre for Mathematical Physics and Stochastics), университет г. Орхус, Дания, 7-12 августа 2003 г., где докладывалась первоначальная версия этой работы.

Автор благодарит О. Джонсона и Ю.М. Сухова за полезное обсуждение окончательного варианта статьи во время визита в колледж St. John's и Статистическую лабораторию Кембриджского университета.

\section{СПИСОК ЛИТЕРАТУРЫ}

1. Хелстром К.У. Квантовая теория проверки гипотез и оценивания. М.: Мир, $1979,344 \mathrm{c}$.

2. Холево A.C. Вероятностные и статистические аспекты квантовой теории. 2-е изд. Москва-Ижевск: ИКИ, 2003, 404 с.

3. Gill R.D., Massar S. State estimation for large ensembles. - Phys. Rev. A, 2000, v. $61,042312 / 1-16$; LANL e-print quant-ph/9902063.

4. Barndorff-Nielsen O. E., Gill R.D., Jupp P.E. On quantum statistical inference. J. Roy. Statist. Soc. B, 2003, v. 65, № 4, p. 775-816.

5. Keyl M., Werner R.F. Estimating the spectrum of a density operator. - Phys. Rev. A(3), 2001, v. 64, № 5, 052311.

6. Hayashi $M$. Asymptotic estimation theory for a finite-dimensional pure state model. - J. Phys. A, 1998, v. 31, № 20, p. 4633-4655. Corrections: 1998, v. 31, № 41, p. 8405 .

7. Hayashi M. Two quantum analogues of Fisher information from a large deviation viewpoint of quantum estimation. - J. Phys. A, 2002, v. 35, № 36, p. 7689-7727.

8. Matsumoto $K$. A new approach to the Cramér-Rao-type bound of the pure-state model. - J. Phys. A, 2002, v. 35, № 13, p. 3111-3123.

9. Холево А.C. Статистическая структура квантовой теории. Москва-Ижевск: ИКИ, 2003, $192 \mathrm{c.}$

10. Рухин А.Л. Некоторые статистические и вероятностные задачи на группах. Труды МИАН, 1970, т. 111, с. 52-109.

11. Линник Ю. В. Теоретико-информационное доказательство центральной предельной теоремы в условиях Линдеберга. - Теория вероятн. и ее примен., 1959, т. 4, B. 3, c. 311-320.

12. Johnson O., Suhov Yu. M. Entropy and random vectors. - J. Statist. Phys., 2001, v. 104, p. $147-167$.

13. Johnson $O$., Barron A. Fisher information inequalities and the central limit theorem. LANL e-reprint math PR/0111020.

14. Holevo A.S. Bounds for generalized uncertainty of shift parameter. - Lecture Notes in Math., 1983, v. 1021, p. 243-251.

15. Холево A.C. Обобщенные системы импримитивности для абелевых групп. Изв. вузов, сер. матем., 1983, № 2, с. 49-71.

16. Прохоров Ю.В. Локальная теорема для плотностей. - Докл. АН СССР, 1952, т. 83 , № 6 , c. 797-800.

17. Крамер Г. Математические методы статистики. М.: Мир, 1975, 648 с. 